\title{
Best Combination of Machine Learning Algorithms for Course Recommendation System in E-learning
}

\author{
Sunita B Aher \\ M.E. (CSE) -II \\ Walchand Institute of Technology \\ Solapur University India
}

\author{
Lobo L.M.R.J. \\ Associate Professor, Head, Department of IT \\ Walchand Institute of Technology \\ Solapur University India
}

\begin{abstract}
Data Mining is the extraction of hidden predictive information from large database which can be used in various commercial applications like bioinformatics, Ecommerce etc. Association Rule, classification and clustering are three different algorithms in data mining. Course Recommender System plays an important role in identifying the behavior of students interested in particular set of courses. We collect the data regarding the course enrollment for specific set of data. For collecting this data, we use the learning management system like Moodle. After collecting the data, we apply the different combination of data mining algorithm like classification \& association rule algorithm, clustering \& association rule algorithm, association rule mining in classified \& clustered data, combining clustering \& classification algorithm in association rule algorithms or simply the association rule algorithm. Here in this paper we use ADTree classification algorithm, Simple K-means Algorithm \& Apriori Association Rule algorithm as different machine learning algorithm. So we propose the five different methods to find the best combination of algorithm in recommending the courses to students in E-learning. We compare the result of this combined approach as well as only the association rule algorithm $\&$ present the best combination of algorithm for recommendation of courses in E-learning according to our simulation.
\end{abstract}

\section{Keywords}

Weka, Machine Learning Algorithm, Simple K-means Algorithm, ADTree Classification Algorithm, Apriori Association Rule Algorithm, Moodle.

\section{INTRODUCTION}

Data mining also known as Knowledge Discovery in Database (KDD) is the extraction of interesting (nontrivial, implicit, previously unknown and potentially useful) patterns or knowledge from huge amount of data. Alternative names to data mining are knowledge extraction, data/pattern analysis, data archeology, data dredging, information harvesting, business intelligence, etc.

Data mining can also be used to extract the knowledge from E-learning system such as Moodle. The Course Recommendation System in E-Learning is a system that recommend to the student, the best combination of courses in which the students are interested.

The rest of the document is organized as follows. Section 1.1 provides a background of the related research fields covering a brief introduction about each. Section 2 describes the Literature Review. Section 3 discusses the architecture of proposed system. Section $4 \& 5$ discusses methodology \& implementation and best combination of data mining algorithm. The conclusion \& future work is presented in Section 6 followed by references.

\subsection{Background}

This research integrates issues from the research field of data mining algorithms such as Classification, clustering \& Association Algorithm), Moodle and open source data mining tool, Weka. The following subsections include a brief overview of these topics.

\subsubsection{Apriori Association Rule Algorithm}

Association rules are used to show the relationship between data items. Association rule generation is usually split up into two separate steps: First, minimum support is applied to find all frequent itemsets in a database. Second, these frequent itemsets and the minimum confidence constraint are used to form rules.

Apriori Association rule is used to mine the frequent patterns in database. Support \& confidence are the normal method used to measure the quality of association rule. Support for the association rule $\mathrm{X}->\mathrm{Y}$ is the percentage of transaction in the database that contains XUY. Confidence for the association rule is $\mathrm{X}->\mathrm{Y}$ is the ratio of the number of transaction that contains XUY to the number of transaction that contain X [9]. The Apriori association rule algorithm is given in below [9]:

Apriori Association Rule Algorithm

Input : Database of Transactions $\mathrm{D}=\left\{\mathrm{t}_{1}, \mathrm{t} 2, \ldots, \mathrm{t}_{\mathrm{n}\}}\right.$ Set if Items $\mathrm{I}=\left\{\mathrm{I}_{1}, \mathrm{I}_{2}, \ldots . . \mathrm{I}_{\mathrm{k}}\right\}$

Frequent (Large) Itemset L

Support,

Confidence.

Output : Association Rule satisfying Support \& Confidence

Method :

$\mathrm{C}_{1}=$ Itemsets of size one in I;

Determine all large itemsets of size $1, \mathrm{~L}_{1}$;

$\mathrm{i}=1$;

Repeat

$\mathrm{i}=\mathrm{i}+1$

$\mathrm{C}_{\mathrm{i}}=$ Apriori-Gen $\left(\mathrm{L}_{\mathrm{i}-1}\right)$

Apriori-Gen $\left(\mathrm{L}_{\mathrm{i}-1}\right)$

1. Generate candidates of size $i+1$ from large itemsets of size $i$

2. Join large itemsets of size $i$ if they agree on i-

3. Prune candidates who have subsets that are not large.

Count $\mathrm{C}_{\mathrm{i}}$ to determine $\mathrm{L}_{\mathrm{i}}$

Until no more large itemsets found; 


\subsubsection{Simple K-means Clustering Algorithms}

Clustering is finding groups of objects such that the objects in one group will be similar to one another and different from the objects in another group [8]. Clustering can be considered the most important unsupervised learning technique.

Simple K-means algorithm is a type of unsupervised algorithm in which items are moved among the set of cluster until required set is reached. This algorithm is used to classify the data set, provided the number of cluster is given in prior. This algorithm is iterative in nature.

\section{Algorithm: Simple K-means clustering algorithm} Input:

Set of Elements or Database of transaction

$$
D=\left\{t_{1}, t_{2}, t_{3}, \ldots, t_{n}\right\}
$$

Output:

Number of required Cluster $\mathrm{k}$

Method:

$$
\text { Set of Cluster K }
$$

Make initial guesses for the means $\mathbf{m}_{\mathbf{1}}, \mathbf{m}_{\mathbf{2}}, \ldots, \mathbf{m}_{\mathbf{k}}$ Repeat

Assign each element $t_{i}$ to the cluster having the closest mean.

Calculate the new mean for each cluster.

Until there are no changes in any mean

\subsubsection{ADTree Classification Algorithm}

Classification is a data mining task that maps the data into predefined groups \& classes. It is also called as supervised learning.

An alternating decision tree (ADTree) is a machine learning method for classification which generalizes decision trees. An alternating decision tree consists of two nodes. Decision nodes specify a predicate condition. Prediction nodes contain a single number. ADTree always have prediction nodes as both root and leaves. An instance is classified by an ADTree by following all paths for which all decision nodes are true and summing any prediction nodes that are traversed.

\subsubsection{Learning Management System Moodle}

Moodle is an open-source course management learning system to help educators create effective online learning communities [15]. It is also possible to modify the source code of any file of Moodle. It is very easy to add the course in the system. Here are adding 13 course category $\&$ near about 82 subjects which are related to Computer Science \& Engineering and Information Technology Department. Here we are using the Moodle as it is very user friendly. Also it maintains detailed logs of all activities of students. It stores the record of every click that students make. We can use these logs to find courses in which student are interested. It stores the logs in relational database MYSQL. Moodle is shown in figure 1.

\subsubsection{Open Source Data Mining Tool Weka}

Weka is a collection of machine learning algorithms for data mining tasks. The algorithms can either be applied directly to a dataset or called from your own Java code [12]. The Weka workbench contains a collection of visualization tools and algorithms for data analysis and predictive modeling, together with graphical user interfaces for easy access to this functionality [11]. It is freely available software. Weka has several standard data mining tasks, data preprocessing, clustering, classification, association, visualization, and feature selection. Figure 2 shows Weka 3.5.3 with Explorer window.

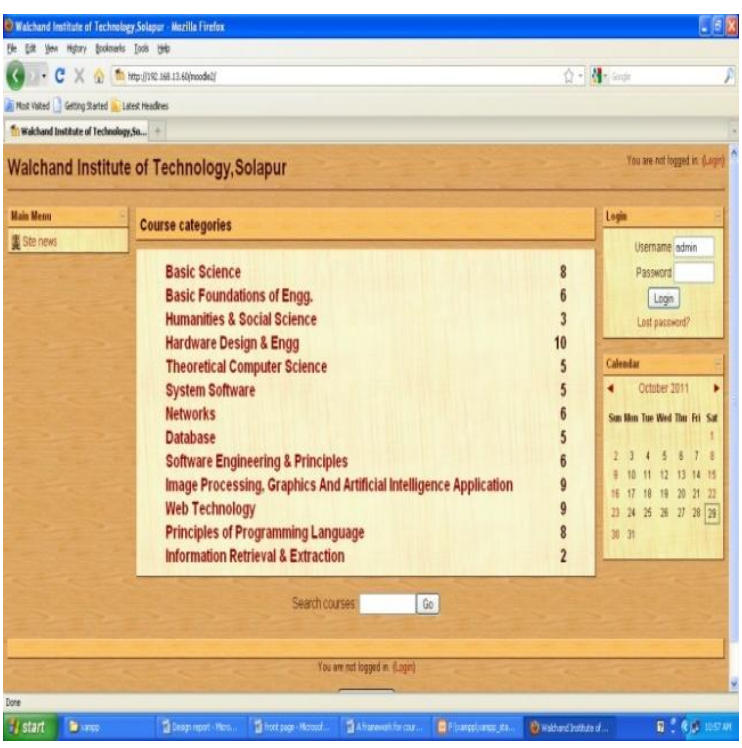

Figure 1: Moodle

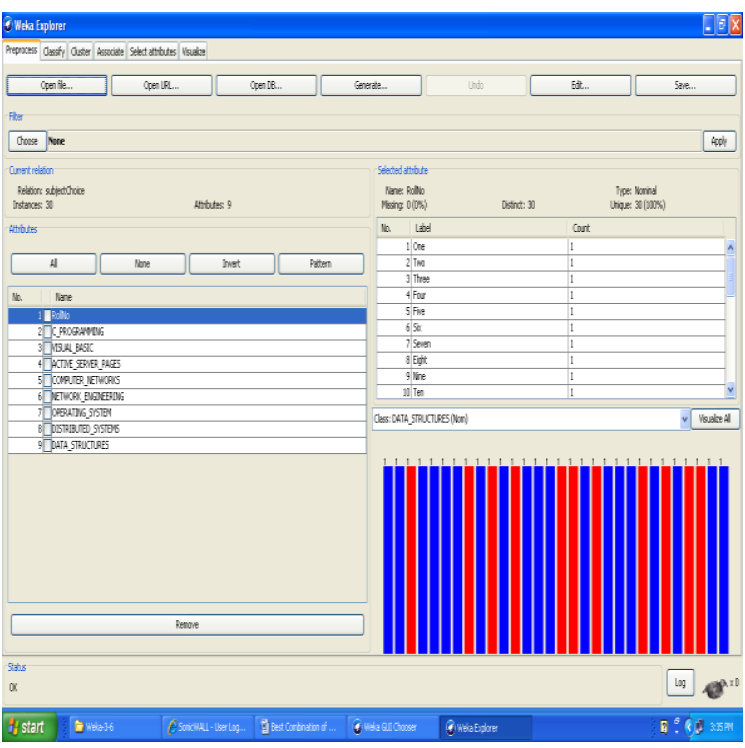

Figure 2: Weka 3.5.3 with Explorer window

\section{LITERATURE REVIEW}

C. Carmona [1], they propose proposed how to use adaptive machine learning algorithms to learn about the student's preferences over time. First they use all the background knowledge available about a particular student to build an initial decision model based on learning styles. This model can then be fine-tuned with the data generated by the student's interactions with the system in order to reflect more accurately his/her current preferences.

Castro [2] provides an up-to-date snapshot of the current state of research and applications of Data mining methods in e-learning. It provides the taxonomy of e-learning problems to which the Data Mining techniques have been applied including, for instance: Students' classification based on their learning performance; detection of irregular learning behaviors; e-learning system navigation and interaction optimization; clustering according to similar elearning system usage; and systems' adaptability to 
students' requirements and capacities.

Lili He \& Hongtao Bai [3] explained Aspect oriented programming which offers a unique module, an aspect, to encapsulate scattered and tangled code, which made it hopeful to solve the problem of crosscutting concerns. Identification and encapsulation of crosscutting concerns was the key problem in the migration from $\mathrm{OO}$ system to AO system. A novel aspect mining method which combined clustering and association rule technology is provided in this article. Clustering analysis based on the execution traces was provided to find out candidate aspects; while association rule mining based on the execution traces with ordered call was used to find out the crosscuts. Both the aspect code (advice body) and the crosscuts (point cuts) were gotten after the above two processes, which constituted the aspect mining process.

Ald[3pio Jorge [4] proposed a method for grouping and summarizing large sets of association rules according to the items contained in each rule. They used hierarchical clustering to partition the initial rule set into thematically coherent subsets. This enabled the summarization of the rule set by adequately choosing a representative rule for each subset, and helped in the interactive exploration of the rule model by the user. Rule clusters can also be used to infer novel interest measures for the rules.

B.Ramasubbareddy, A. Govardhan \& A.Ramamohanreddy [13] proposed Associative classification which was a classification of a new tuple using association rules. It was a combination of association rule mining and classification. They searched for strong associations between frequent patterns and class labels. The main aim of this paper was to improve accuracy of a classifier. The accuracy can be achieved by producing all types of negative class association rules.

The main hypothesis discussed in the paper [4] was that Web content analysis can be used to improve Web usage mining results. They proposed a system that integrated Web page clustering into log file association mining and used the cluster labels as Web page content indicators. They experimented with several approaches to content clustering, relying on keyword and character n-gram based clustering with different distance measures and parameter settings.

\section{ARCHITECTURE OF COURSE RECOMMENDER SYSTEM}

In Course Recommendation System, we are consider the 13 course category which is shown in following table 1 . Under each category there will courses. So there are about 82 subjects.

We have created the student login \& gave the access to the student. We have considered the student of two courses Computer Science \& Engineering and Information Technology for collecting the data. Student will enroll for those subjects in which they are interested. This data is stored in the moodle database which we use to find out the best combination. The framework for this recommendation system is shown in the figure 3 .

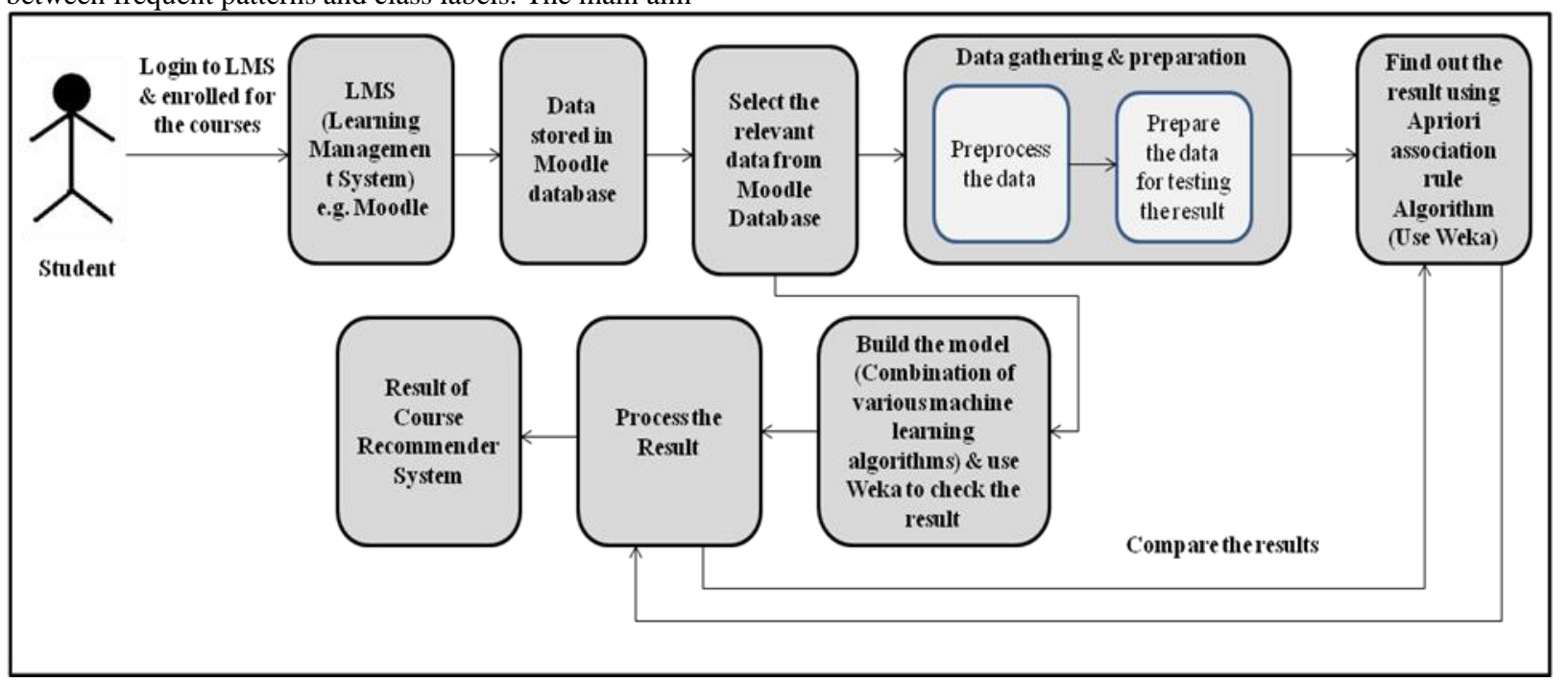

Figure 3: Course Recommender System in E-learning

In this architecture, student first logs in the Learning Management System e.g. Moodle. The system verifies the username \& password. After verifying the username \& password, student will search the course category \& courses (subjects). Students will enroll for subject in which they are interested. This enrollment information is stored in database as Moodle store each \& every action of student.

After collecting the data from student which is stored in Moodle database, the next stage is to gather \& prepare the data. In this step, first we select the data from database which is relevant. We check the result using the Apriori association rule algorithm. After that we clean \& transformed into the format for testing. To test the result we use the open source data mining tool WEKA. Since our project is to find the best combination of subject, we use the Apriori association rule algorithm. In the step, build the model; we try to develop the algorithm which may be combination of various data mining algorithm. We find out the result using this model \& compare the result with result obtained using already existing algorithm in WEKA. Finally we deploy the result.

\section{METHODOLOGY\& MPLEMENTATION RESULT}

Here we are considering the sample data extracted from Moodle database. Here we consider data of 15 courses. We consider the subjects like C-programming (C), Visual 
Basic (VB), Active Server Pages (ASP), Computer Network (CN), Microprocessor (MP), Computer Organization (CO), Database Engineering (DBE), Advanced Database System (ADS), Operating System
(OS), Distributed System (DS), Finite Automata System (FSA), Data Structure (DS-I), Software Engineering (SE), and Software Testing \& Quality assurance (STQA).

Table 1: Sample data extracted from Moodle database [11]

\begin{tabular}{|c|c|c|c|c|c|c|c|c|c|c|c|c|c|c|c|}
\hline $\begin{array}{c}\text { Courses } \rightarrow \\
\text { Roll No } \downarrow\end{array}$ & $\mathrm{C}$ & VB & ASP & $\mathrm{CN}$ & $\mathrm{NE}$ & MP & $\mathrm{CO}$ & DBE & ADS & OS & DS & FSA & $\begin{array}{l}\text { DS- } \\
\text { I }\end{array}$ & SE & STQA \\
\hline 1 & yes & yes & yes & yes & yes & no & no & no & no & no & no & no & yes & no & no \\
\hline 2 & no & no & no & no & no & no & no & no & no & no & no & no & no & no & no \\
\hline 3 & yes & yes & yes & yes & yes & no & no & no & no & yes & yes & yes & yes & yes & yes \\
\hline 4 & no & no & no & yes & yes & no & yes & no & no & no & no & no & no & no & no \\
\hline 5 & yes & yes & yes & yes & yes & no & no & yes & no & yes & yes & no & yes & no & no \\
\hline 6 & yes & yes & yes & no & no & no & no & no & no & yes & no & no & yes & no & no \\
\hline 7 & no & no & no & yes & yes & yes & yes & no & no & no & no & no & no & yes & no \\
\hline 8 & no & no & no & no & no & no & no & yes & yes & yes & yes & no & yes & no & no \\
\hline 9 & no & no & no & yes & yes & yes & yes & no & no & no & no & yes & no & no & no \\
\hline 10 & yes & no & no & no & no & no & no & no & no & no & no & no & no & no & no \\
\hline 11 & yes & yes & yes & no & no & no & no & no & no & yes & yes & no & yes & no & no \\
\hline 12 & yes & yes & yes & yes & yes & no & no & no & no & no & no & no & no & no & no \\
\hline 13 & no & no & no & no & no & no & no & yes & yes & yes & yes & no & yes & yes & yes \\
\hline 14 & yes & yes & yes & yes & yes & no & no & no & no & yes & yes & no & no & no & no \\
\hline 15 & yes & yes & yes & no & no & no & no & no & no & no & no & no & yes & no & no \\
\hline 16 & no & no & no & yes & yes & no & no & yes & yes & yes & yes & no & yes & no & no \\
\hline 17 & yes & yes & yes & no & no & no & no & no & no & yes & yes & no & yes & yes & yes \\
\hline 18 & yes & yes & yes & no & no & no & no & no & no & no & no & no & no & no & no \\
\hline 19 & no & no & no & yes & yes & yes & yes & yes & yes & no & no & no & no & no & no \\
\hline 20 & yes & no & no & no & no & no & no & no & no & yes & yes & no & yes & yes & yes \\
\hline 21 & yes & no & yes & no & no & yes & yes & no & no & yes & yes & yes & no & no & no \\
\hline 22 & no & no & no & no & no & no & no & yes & yes & yes & yes & no & yes & no & no \\
\hline 23 & yes & yes & yes & yes & yes & yes & yes & no & no & yes & yes & no & yes & no & no \\
\hline 24 & yes & yes & yes & yes & yes & yes & yes & yes & yes & yes & yes & yes & yes & yes & yes \\
\hline 25 & no & yes & yes & no & no & yes & yes & yes & yes & yes & yes & no & no & no & no \\
\hline 26 & yes & yes & yes & no & no & no & no & no & no & yes & yes & no & yes & no & no \\
\hline 27 & yes & yes & yes & yes & yes & no & no & no & no & no & no & no & no & no & no \\
\hline 28 & no & no & no & yes & yes & no & no & no & no & yes & yes & no & yes & no & no \\
\hline 29 & no & no & no & no & no & yes & yes & yes & yes & no & no & no & no & no & no \\
\hline 30 & yes & yes & yes & yes & yes & no & no & no & no & no & no & no & no & yes & yes \\
\hline 31 & no & no & no & no & no & no & no & no & no & no & no & no & no & no & no \\
\hline 32 & yes & yes & yes & no & no & no & no & yes & yes & yes & yes & no & yes & no & no \\
\hline 33 & no & no & no & yes & yes & no & no & no & no & yes & yes & no & yes & no & no \\
\hline 34 & yes & yes & yes & no & no & no & no & no & no & no & no & no & no & no & no \\
\hline 35 & no & no & no & no & no & no & no & no & no & yes & yes & no & no & no & no \\
\hline 36 & no & no & no & yes & yes & no & no & no & no & no & no & no & yes & no & no \\
\hline 37 & yes & yes & yes & yes & yes & yes & yes & yes & yes & no & no & no & no & no & no \\
\hline 38 & no & no & no & no & no & no & no & no & no & yes & yes & yes & yes & yes & yes \\
\hline 39 & yes & yes & yes & yes & yes & yes & yes & yes & yes & yes & yes & yes & yes & yes & yes \\
\hline 40 & no & no & no & no & no & no & no & no & no & no & no & no & no & yes & yes \\
\hline 41 & yes & yes & yes & no & no & no & no & no & no & yes & yes & no & yes & no & no \\
\hline 42 & no & no & no & yes & yes & no & no & no & no & no & no & no & no & no & no \\
\hline 43 & no & no & no & no & no & no & no & no & no & yes & yes & no & yes & no & no \\
\hline 44 & no & no & no & no & no & no & no & no & no & no & no & no & no & no & yes \\
\hline 45 & no & no & no & no & no & no & no & no & no & no & no & no & no & no & no \\
\hline
\end{tabular}
association rules containing "yes" only. So in order to get the correct association rule, we prepare the data

\subsection{Association Rule Mining}

If we apply Apriori association rule mining to the data extracted from Moodle database using data mining tool Weka then we get all association rule containing "no"

only. This result is shown in first row of table 7. As we are recommending the course to the student, we need all

\subsubsection{Data Preparation Strategy}

Data preparation strategy is explained in [10]. For data preparation, we delete those rows having very less student count as well as that course having very less course count. 
Table 2: Table 1 after data preparation step [11]

\begin{tabular}{|c|c|c|c|c|c|c|c|c|}
\hline $\begin{array}{l}\text { Subject } \\
\text { Roll } \\
\text { No of } \\
\text { Student } \\
\quad \downarrow\end{array}$ & $\mathrm{C}$ & VB & $\begin{array}{l}\text { AS } \\
P\end{array}$ & $\mathrm{CN}$ & $\mathrm{NE}$ & OS & DS & $\begin{array}{l}\text { DS } \\
\text {-I }\end{array}$ \\
\hline 1 & yes & yes & yes & yes & yes & no & no & yes \\
\hline 3 & yes & yes & yes & yes & yes & yes & yes & yes \\
\hline 4 & no & no & no & yes & yes & no & no & no \\
\hline 5 & yes & yes & yes & yes & yes & yes & yes & yes \\
\hline 6 & yes & yes & yes & no & no & yes & no & yes \\
\hline 7 & no & no & no & yes & yes & no & no & no \\
\hline 8 & no & no & no & no & no & yes & yes & yes \\
\hline 9 & no & no & no & yes & yes & no & no & no \\
\hline 11 & yes & yes & yes & no & no & yes & yes & yes \\
\hline 12 & yes & yes & yes & yes & yes & no & no & no \\
\hline 13 & no & no & no & no & no & yes & yes & yes \\
\hline 14 & yes & yes & yes & yes & yes & yes & yes & no \\
\hline 15 & yes & yes & yes & no & no & no & no & yes \\
\hline 16 & no & no & no & yes & yes & yes & yes & yes \\
\hline 17 & yes & yes & yes & no & no & yes & yes & yes \\
\hline 18 & yes & yes & yes & no & no & no & no & no \\
\hline 19 & no & no & no & yes & yes & no & no & no \\
\hline 20 & yes & no & no & no & no & yes & yes & yes \\
\hline 21 & yes & no & yes & no & no & yes & yes & no \\
\hline 22 & no & no & no & no & no & yes & yes & yes \\
\hline 23 & yes & yes & yes & yes & yes & yes & yes & yes \\
\hline 24 & yes & yes & yes & yes & yes & yes & yes & yes \\
\hline 25 & no & yes & yes & no & no & yes & yes & no \\
\hline 26 & yes & yes & yes & no & no & yes & yes & yes \\
\hline 27 & yes & yes & yes & yes & yes & no & no & no \\
\hline 28 & no & no & no & yes & yes & yes & yes & yes \\
\hline 30 & yes & yes & yes & yes & yes & no & no & no \\
\hline 32 & yes & yes & yes & no & no & yes & yes & yes \\
\hline 33 & no & no & no & yes & yes & yes & yes & yes \\
\hline 34 & yes & yes & yes & no & no & no & no & no \\
\hline 35 & no & no & no & no & no & yes & yes & no \\
\hline 36 & no & no & no & yes & yes & no & no & yes \\
\hline
\end{tabular}

\begin{tabular}{|l|l|l|l|l|l|l|l|l|}
\hline 37 & yes & yes & yes & yes & yes & no & no & no \\
\hline 38 & no & no & no & no & no & yes & yes & yes \\
\hline 39 & yes & yes & yes & yes & yes & yes & yes & yes \\
\hline 41 & yes & yes & yes & no & no & yes & yes & yes \\
\hline 42 & no & no & no & yes & yes & no & no & no \\
\hline 43 & no & no & no & no & no & yes & yes & yes \\
\hline
\end{tabular}

After data preparation strategy, we get table 2. After preparation of data, we check the result using Weka. These results are shown in second row of table 7 . Now, we get all association rules containing "yes" only. As we increase the support, we get the refined rule. The meaning of the rule (DS=yes $\rightarrow \mathrm{OS}=$ yes) is: if student is interested in Distributed system then he/she is interested in Operating System \& vice versa. Due to above rule, we can recommend to new student who has recently enrolled for DS course, the Operating System as a course to be opted. Association rule obtained here also match with the in general real world interdependencies among the course.

\subsection{Combination of Classification \& Association Rule algorithm}

Here we consider the ADTree algorithm as classification algorithm \& Apriori Rule as association rule algorithm. First we apply the ADTree classification algorithm on sample data. We consider only those courses for which the value of ADTree is negative (negative value represent "yes"). Then we select those rows from table having the negative course value. The result after application of ADTree classification algorithm in table 1 is shown in table 3. Now we apply the Apriori association rule algorithm to this table. The result of application of this algorithm is shown in third row of table 7. Using this combination of classification \& association rule algorithm, there is no need to prepare the data obtained from Moodle courses.

Table 3: After application of ADTree classification algorithm on table 2 [17]

\begin{tabular}{|r|l|l|l|l|l|l|l|l|l|l|l|l|l|l|l|}
\hline Courses $\rightarrow$ & C & VB & ASP & CN & NE & MP & CO & DBE & ADS & OS & DS & FSA & DS-I & SE & STQA \\
\hline & & & & & & & & & & & & & & & \\
\hline 3 & yes & yes & yes & yes & yes & no & no & no & no & yes & yes & yes & yes & yes & yes \\
\hline 5 & yes & yes & yes & yes & yes & no & no & yes & no & yes & yes & no & yes & no & no \\
\hline 11 & yes & yes & yes & no & no & no & no & no & no & yes & yes & no & yes & no & no \\
\hline 17 & yes & yes & yes & no & no & no & no & no & no & yes & yes & no & yes & yes & yes \\
\hline 20 & yes & no & no & no & no & no & no & no & no & yes & yes & no & yes & yes & yes \\
\hline 23 & yes & yes & yes & yes & yes & yes & yes & no & no & yes & yes & no & yes & no & no \\
\hline 24 & yes & yes & yes & yes & yes & yes & yes & yes & yes & yes & yes & yes & yes & yes & yes \\
\hline 26 & yes & yes & yes & no & no & no & no & no & no & yes & yes & no & yes & no & no \\
\hline 32 & yes & yes & yes & no & no & no & no & yes & yes & yes & yes & no & yes & no & no \\
\hline 39 & yes & yes & yes & yes & yes & yes & yes & yes & yes & yes & yes & yes & yes & yes & yes \\
\hline 41 & yes & yes & yes & no & no & no & no & no & no & yes & yes & no & yes & no & no \\
\hline
\end{tabular}

\subsection{Combination of Clustering \& Association Rule Algorithm}

In this combination of algorithms i.e. Simple K-means clustering algorithm \& Apriori association rule algorithm, first we apply the Simple K-means clustering algorithm to sample data extracted from Moodle course. After application of this algorithm we get some clusters. So we divide the table into sub tables depending on the number of clusters. Then we check the result of these sub-tables. So we consider only that sub-table of cluster which gives the correct result. The cluster which gives the correct result is shown in table 4. As this is Course Recommending System, we consider only that association rule containing "yes" only. After clustering the data using Simple Kmeans clustering algorithm, we apply the Apriori 
association rule algorithm to the sub-table whose result are correct. The result of application of Apriori to clustered

data is shown in forth row of table 7.

Table 4: After application of Simple K-means clustering algorithm on table 2 [19]

\begin{tabular}{|c|c|c|c|c|c|c|c|c|c|c|c|c|c|c|c|}
\hline $\begin{array}{c}\text { Courses } \rightarrow \\
\text { Roll No } \downarrow\end{array}$ & $\mathrm{C}$ & VB & ASP & $\overline{\mathrm{CN}}$ & $\mathrm{NE}$ & MP & $\mathrm{CO}$ & $\overline{D B E}$ & ADS & OS & DS & FSA & DS-I & $\mathrm{SE}$ & STQA \\
\hline 1 & yes & yes & yes & yes & yes & no & no & no & no & no & no & no & yes & no & no \\
\hline 3 & yes & yes & yes & yes & yes & no & no & no & no & yes & yes & yes & yes & yes & yes \\
\hline 5 & yes & yes & yes & yes & yes & no & no & yes & no & yes & yes & no & yes & no & no \\
\hline 6 & yes & yes & yes & no & no & no & no & no & no & yes & no & no & yes & no & no \\
\hline 11 & yes & yes & yes & no & no & no & no & no & no & yes & yes & no & yes & no & no \\
\hline 12 & yes & yes & yes & yes & yes & no & no & no & no & no & no & no & no & no & no \\
\hline 14 & yes & yes & yes & yes & yes & no & no & no & no & yes & yes & no & no & no & no \\
\hline 16 & no & no & no & yes & yes & no & no & yes & yes & yes & yes & no & yes & no & no \\
\hline 17 & yes & yes & yes & no & no & no & no & no & no & yes & yes & no & yes & yes & yes \\
\hline 23 & yes & yes & yes & yes & yes & yes & yes & no & no & yes & yes & no & yes & no & no \\
\hline 24 & yes & yes & yes & yes & yes & yes & yes & yes & yes & yes & yes & yes & yes & yes & yes \\
\hline 26 & yes & yes & yes & no & no & no & no & no & no & yes & yes & no & yes & no & no \\
\hline 27 & yes & yes & yes & yes & yes & no & no & no & no & no & no & no & no & no & no \\
\hline 28 & no & no & no & yes & yes & no & no & no & no & yes & yes & no & yes & no & no \\
\hline 30 & yes & yes & yes & yes & yes & no & no & no & no & no & no & no & no & yes & yes \\
\hline 32 & yes & yes & yes & no & no & no & no & yes & yes & yes & yes & no & yes & no & no \\
\hline 33 & no & no & no & yes & yes & no & no & no & no & yes & yes & no & yes & no & no \\
\hline 37 & yes & yes & yes & yes & yes & yes & yes & yes & yes & no & no & no & no & no & no \\
\hline 39 & yes & yes & yes & yes & yes & yes & yes & yes & yes & yes & yes & yes & yes & yes & yes \\
\hline 41 & yes & yes & yes & no & no & no & no & no & no & yes & yes & no & yes & no & no \\
\hline
\end{tabular}

\subsection{Association Rule Mining of classified \& clustered data}

In this case we consider three algorithms i.e. ADTree classification algorithm, Simple K-means clustering algorithm \& Apriori Association Rule algorithm. The order of application of these three algorithms is:

ADTree classification algorithm- Simple K-means clustering algorithm- Apriori association rule algorithm
So first we apply the ADTree classification algorithm on sample table of moodle course. Then we apply Simple Kmeans clustering algorithm on classified data. Again we consider that cluster which gives the correct association rules. Sample table after application of Simple K-means clustering algorithm on classified data which is shown in table 3 is given in table 5. Result of application of Apriori association rule on correct cluster of classified data is shown in fifth row of table 7.

Table 5: After application of Simple K-means clustering algorithm on table 3 [16]

\begin{tabular}{|r|l|l|l|l|l|l|l|l|l|l|l|l|l|l|l|}
\hline $\begin{array}{l}\text { Courses } \rightarrow \\
\text { Roll_No } \downarrow\end{array}$ & C & VB & ASP & CN & NE & MP & CO & DBE & ADS & OS & DS & FSA & $\begin{array}{l}\text { DS- } \\
\text { I }\end{array}$ & SE & $\begin{array}{l}\text { ST } \\
\text { QA }\end{array}$ \\
\hline 5 & yes & yes & yes & yes & yes & no & no & yes & no & yes & yes & no & yes & no & no \\
\hline 11 & yes & yes & yes & no & no & no & no & no & no & yes & yes & no & yes & no & no \\
\hline 23 & yes & yes & yes & yes & yes & yes & yes & no & no & yes & yes & no & yes & no & no \\
\hline 26 & yes & yes & yes & no & no & no & no & no & no & yes & yes & no & yes & no & no \\
\hline 32 & yes & yes & yes & no & no & no & no & yes & yes & yes & yes & no & yes & no & no \\
\hline 41 & yes & yes & yes & no & no & no & no & no & no & yes & yes & no & yes & no & no \\
\hline
\end{tabular}

\subsection{Combining Clustering \& Classification algorithm with Association Rule algorithm}

Here we apply three data mining algorithm in following order:

Simple K-means clustering algorithm- ADTree classification algorithm- ADTree classification algorithm sample data. After clustering the data, we apply the ADTree classification algorithm on clustered data. Sample table after application of ADTree classification algorithm on clustered data which is shown in table 4 is given in table 6. After application of this classification algorithm, we apply Apriori association rule algorithm to classified data whose results are shown in sixth row of table 7 .

First we apply Simple K-means clustering algorithm on 
Table 6: After application of ADTree classification algorithm on table 2 [18]

\begin{tabular}{|r|l|l|l|l|l|l|l|l|l|l|l|l|l|l|l|}
\hline Courses $\rightarrow$ & C & VB & ASP & CN & NE & MP & CO & DBE & ADS & OS & DS & FSA & DS-I & SE & STQA \\
Roll_No $\downarrow$ & & & & & & & & & & & & & & & \\
\hline 3 & yes & yes & yes & yes & yes & no & no & no & no & yes & yes & yes & yes & yes & yes \\
\hline 5 & yes & yes & yes & yes & yes & no & no & yes & no & yes & yes & no & yes & no & no \\
\hline 23 & yes & yes & yes & yes & yes & yes & yes & no & no & yes & yes & no & yes & no & no \\
\hline 24 & yes & yes & yes & yes & yes & yes & yes & yes & yes & yes & yes & yes & yes & yes & yes \\
\hline 39 & yes & yes & yes & yes & yes & yes & yes & yes & yes & yes & yes & yes & yes & yes & yes \\
\hline
\end{tabular}

\section{BEST COMBINATION OF DATA MINING ALGORITHM}

According to our simulation, the best combination of algorithm is the combination of clustering, classification \& association rule mining. Here we preprocess the data to remove the noise or to add the missing value but there is no need to prepare the data as explained subsection 4.1.1. If we are using the Apriori association rule algorithm \& increase the support then we are getting the refined association rule but the number of rules, we get, are less \& we need to prepare the data also. Association rule obtained using this combination i.e. clustering, classification \& association rule algorithm also match with the in general real world interdependencies among the courses.

Figure 4 shows the graph for courses after application of data mining techniques

Table 7: Result of applying the different data mining technique

\begin{tabular}{|c|c|}
\hline $\begin{array}{l}\text { Course } \\
\text { considered }\end{array}$ & Results \\
\hline \multicolumn{2}{|r|}{$\begin{array}{c}\text { Result of Apriori Association Rule before preprocessing \& application of combination of Clustering \& Association } \\
\text { Rule }\end{array}$} \\
\hline $\begin{array}{lr}\text { C, } & \text { VB, } \\
\text { ASP, } & \text { CN, } \\
\text { NE, } & \text { MP, } \\
\text { CO, } & \text { DBE, } \\
\text { ADS, OS, } & \text { OS, FSA, } \\
\text { DS, } & \text { SS-I, SE, } \\
\text { STQA }\end{array}$ & $\begin{array}{l}\text { Minimum support: } 0.7 \\
\text { Minimum metric <confidence>: } 0.9 \\
\text { Best rules found: } \\
\text { 1. Computer_Organization=no } \rightarrow \text { Microprocessor=no conf:(1) } \\
\text { 2. Database_Engineering=no } \rightarrow \text { Advanced_Database_System }=\text { no conf: }(1) \\
\text { 3. Computer_Organization=no Finite_State_Automata=no } 32 \rightarrow \text { Microprocessor=no conf:(1) } \\
\text { 4. Microprocessor=no } \rightarrow \text { Computer_Organization=no conf: }(0.97) \\
\text { 5. Softare_Tesing_And_Quality_Assurance=no } 35 \rightarrow \text { Software_Engineering=no conf:(0.97) } \\
\text { 6. Software_Engineering=no } \rightarrow \text { Softare_Tesing_And_Quality_Assurance=no conf: }(0.97) \\
\text { 7. Advanced_Database_System=no } \rightarrow \text { Database_Engineering=no conf: }(0.97) \\
\text { 8. Microprocessor=no Finite_State_Automata=no } \rightarrow \text { Computer_Organization=no conf:(0.97) } \\
\text { 9. Finite_State_Automata=no Softare_Tesing_And_Quality_Assurance=no } \rightarrow \text { Software_Engineering=no } \\
\text { conf:(0.97) } \\
\text { 10. Finite_State_Automata=no Software_Engineering=no } \rightarrow \text { Softare_Tesing_And_Quality_Assurance=no } \\
\text { conf: }(0.97)\end{array}$ \\
\hline \multicolumn{2}{|r|}{$\begin{array}{c}\text { Result of Apriori Association Rule after preprocessing \& before application of combination of Clustering \& } \\
\text { Association Rule }\end{array}$} \\
\hline $\begin{array}{l}\mathrm{C}, \mathrm{VB}, \\
\mathrm{ASP}, \mathrm{CN}, \\
\mathrm{NE}, \mathrm{OS}, \\
\mathrm{DS}, \mathrm{DS}-\mathrm{I}\end{array}$ & $\begin{array}{l}\text { Minimum support: } 0.5 \\
\text { Minimum metric <confidence>: } 0.9 \\
\text { Best rules found: } \\
\text { 1. Distributed_Systems=yes } \rightarrow \text { Operating_System=yes conf:(1) } \\
\text { 2. Visual_Basic=yes } \rightarrow \text { Active_Server_Pages=yes conf:(1) } \\
\text { 3. Network_Engineering=yes } \rightarrow \text { Computer_Networks=yes conf:(1) } \\
\text { 4. Computer_Networks=yes } \rightarrow \text { Network_Engineering=yes conf:(1) } \\
\text { 5. C_Programming=yes Visual_Basic=yes } \rightarrow \text { Active_Server_Pages=yes conf:(1) } \\
\text { 6. Distributed_Systems=yes Data_Structures=yes } \rightarrow \text { Operating_System=yes conf:(1) } \\
\text { 7. Operating_System=yes } \rightarrow \text { Distributed_Systems=yes conf:(0.96) } \\
\text { 8. Active_Server_Pages=yes } \rightarrow \text { C_Programming=yes conf: }(0.96) \\
\text { 9. C_Programming=yes } \rightarrow \text { Active_Server_Pages=yes conf: }(0.96)\end{array}$ \\
\hline
\end{tabular}




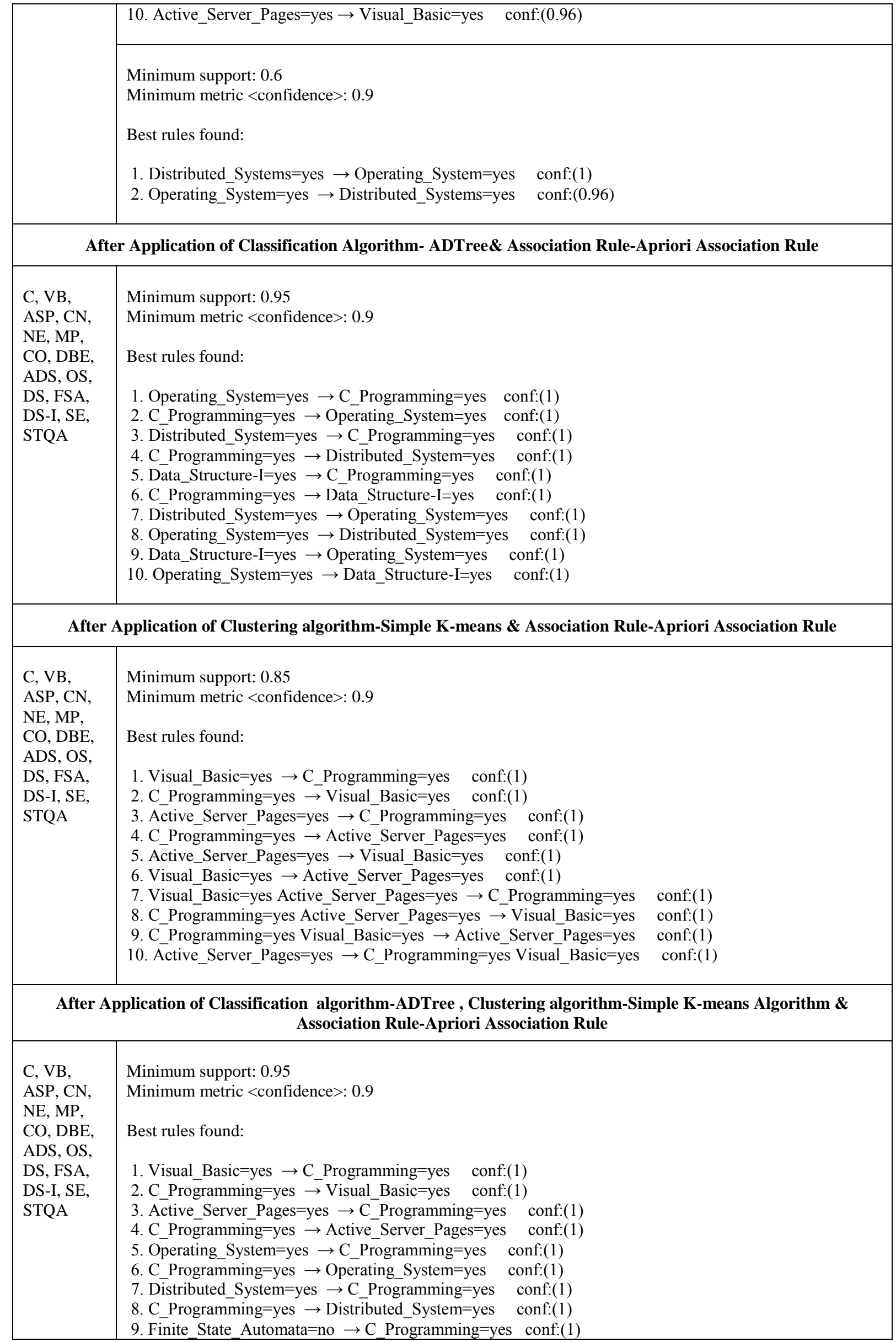




\begin{tabular}{|c|c|}
\hline & 10. C_Programming=yes $\rightarrow$ Finite_State_Automata $=$ no $\quad$ conf:(1) \\
\hline \multicolumn{2}{|c|}{$\begin{array}{c}\text { After Application of Clustering algorithm-Simple K-means, Classification Algorithm- ADTree \& Association Rule- } \\
\text { Apriori Association Rule }\end{array}$} \\
\hline $\begin{array}{l}\mathrm{C}, \mathrm{VB}, \\
\mathrm{ASP}, \mathrm{CN}, \\
\mathrm{NE}, \mathrm{MP}, \\
\mathrm{CO}, \mathrm{DBE}, \\
\text { ADS, OS, } \\
\text { DS, FSA, } \\
\text { DS-I, SE, } \\
\text { STQA }\end{array}$ & $\begin{array}{l}\text { Minimum support: } 0.95 \\
\text { Minimum metric <confidence>: } 0.9 \\
\text { Best rules found: } \\
\text { 1. Visual_Basic=yes } \rightarrow \text { C_Programming=yes conf:(1) } \\
\text { 2. C_Programming=yes } \rightarrow \text { Visual_Basic=yes conf:(1) } \\
\text { 3. Active_Server_Pages=yes } \rightarrow \text { C_Programming=yes conf:(1) } \\
\text { 4. C_Programming=yes } \rightarrow \text { Active_Server_Pages=yes conf:(1) } \\
\text { 5. Computer_Network=yes } \rightarrow \text { C_Programming=yes conf:(1) } \\
\text { 6. C_Programming=yes } \rightarrow \text { Computer_Network=yes conf:(1) } \\
\text { 7. Network_Engineering=yes } \rightarrow \text { C_Programming=yes conf:(1) } \\
\text { 8. C_Programming=yes } \rightarrow \text { Network_Engineering=yes conf:(1) } \\
\text { 9. Operating_System=yes } \rightarrow \text { C_Programming=yes conf:(1) } \\
\text { 10. C_Programming=yes } \rightarrow \text { Operating_System=yes conf:(1) }\end{array}$ \\
\hline
\end{tabular}

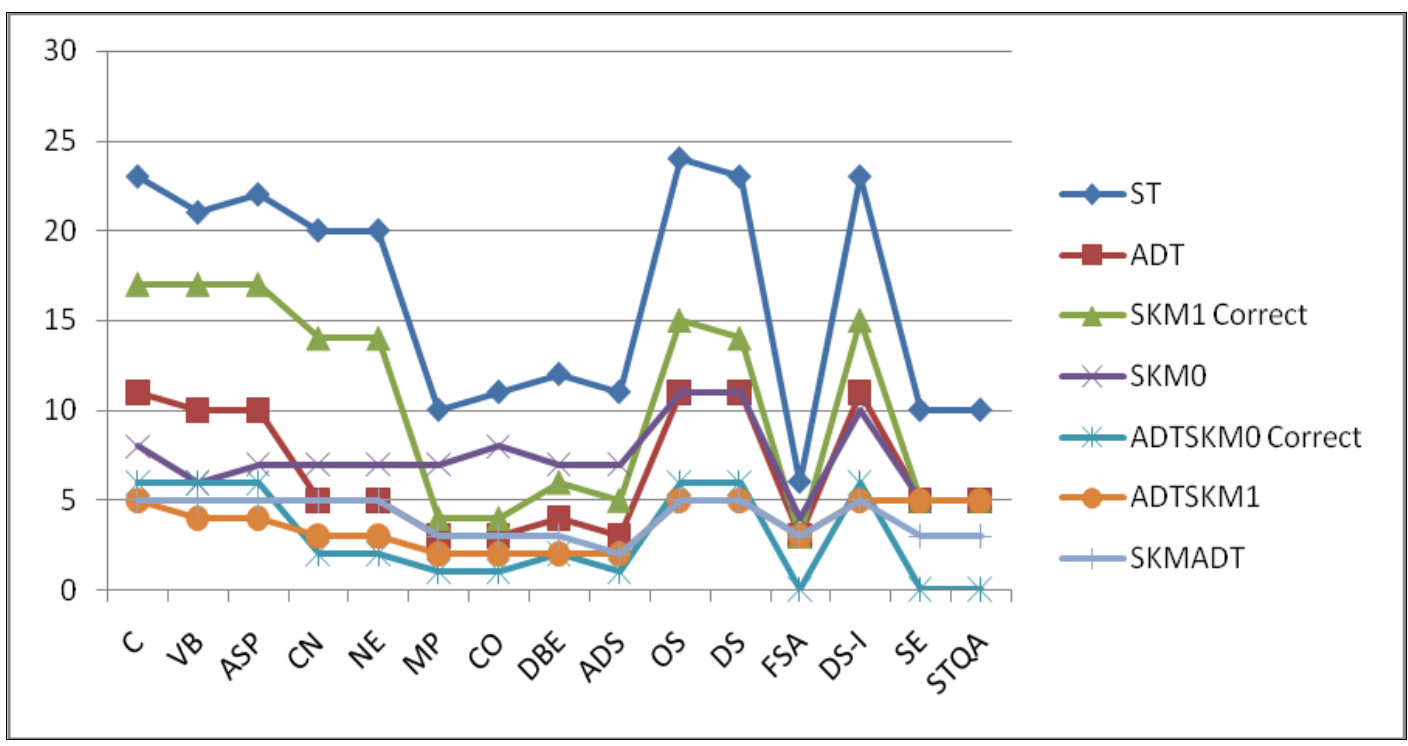

Figure 4 Graph for courses after application of various data mining algorithms

ADT- Courses after application ADTree classification Algorithm to Sample Table

SKM1 Correct - Courses after application of Simple K-means clustering algorithm (cluster 1 correct result) SKM0 - Courses after application of Simple K-means clustering algorithm (cluster 0 incorrect result)

ADTSKM0 Correct - Courses after application of Simple K-means algorithm to classified data (Cluster 0 correct result) ADTSKM0 Correct - Courses after application of Simple K-means algorithm to classified data (Cluster 0 correct result) SKMADT- courses after application of ADTree classification algorithm to correct (SKM1 Correct) clustered data.

\section{CONCLUSION AND FUTURE}

\section{WORK}

In this paper, we compare different combination of data mining algorithms i.e. Classification \& association rule algorithm, clustering \& association rule algorithm, Association Rule Mining of classified \& clustered data, combining Clustering \& Classification algorithm into Association Rule algorithm \& only association rule algorithms. We consider ADTree classification algorithm, Simple K-means clustering algorithm \& Apriori association rule algorithm. We compare the result $\&$ found that the combination clustering, classification \& association rule algorithm is the best combination. Future work includes the atomization of this combination algorithm i.e. clustering, classification \& association rule algorithm on huge amount of data obtained from Moodle course of a college. 


\section{REFERENCES}

[1] C. Carmona, G. Castillo and E. Millán: Discovering Student Preferences in E-learning, EC-TEL07, pp.3342 (2007)

[2] Castro, F., Vellido, A., Nebot, A., \& Mugica, F. (in press). Applying data mining techniques to e-learning problems: A survey and state of the art. In L. C. Jain, R. Tedman, \& D. Tedman (Eds.), Evolution of Teaching and learning paradigms in intelligent environment. Studies in Computational Intelligence (Vol. 62). Springer-Verlag.

[3] Lili He, Hongtao Bai:"Aspect Mining Using Clustering and Association Rule Method" IJCSNS International Journal of Computer Science and Network Security, VOL.6 No.2A, February 2006

[4] Alq33pio Jorge:"Hierarchical Clustering for thematic browsing and summarization of large sets of Association Rules" Supported by the POSI/SRI/39630/2001/Class Project

[6] Sunita B Aher and Lobo L.M.R.J.. Data Mining in Educational System using WEKA. IJCA Proceedings on International Conference on Emerging Technology Trends (ICETT) (3):20-25, 2011. Published by Foundation of Computer Science, New York, USA ISSN $0975-8887$

[7] Han,J. and Kamber, M., "Data Mining: Concepts and Techniques", $2^{\text {nd }}$ edition.

[8] Alaa el-Halees (2009) :"Mining Students Data to Analyze e-Learning Behavior: A Case Study." Accessed from http://uqu.edu.sa/files2/tiny_mce/plugins/filemanager/ files/30/papers/f158.pdf on 05-03-2012

[9] "Data Mining Introductory and Advanced Topics" by Margaret H. Dunham

[10] Sunita B Aher and Lobo L.M.R.J.:" Data Preparation Strategy in E-Learning System using Association Rule Algorithm" selected in International Journal of Computer Applications. ISSN 0975 - 8887

[11] Sunita B Aher and Lobo L.M.R.J. Article: A Framework for Recommendation of courses in Elearning System. International Journal of Computer Applications 35(4):21-28, December 2011. Published by Foundation of Computer Science, New York, USA ISSN $0975-8887$
[12] Weka (2007). http://www.cs.waikato.ac.nz/ml/weka/.

[13] B.Ramasubbareddy, A.Govardhan \& A.Ramamohanreddy:"Classification Based on Positive and Negative Association Rules" International Journal of Data Engineering, (IJDE), Volume (2): Issue (2) : 201184

[14] Jiayun Guo, Vlado Ke`selj, and Qigang Gao:"Integrating Web Content Clustering into Web Log Association Rule Mining?" supported by NSERC

[15] C. Romero, S. Ventura and E. Garcia. Data Mining in Course Management Systems: MOODLE Case Study and Tutorial. Computers and Education, 2007. Num. 51. pp. 368-384.

[16] Sunita B Aher and Lobo L.M.R.J.:" Association Rule Mining of Classified and Clustered Data of ELearning System" selected in ICWET-2012, paper will published in International Journal of Computer Applications (IJCA) ISSN : 0975 - 8887

[17] Sunita B Aher and Lobo L.M.R.J. Article: Mining Association Rule in Classified Data for Course Recommender System in E-Learning. International Journal of Computer Applications 39(7):1-7, February 2012. Published by Foundation of Computer Science, New York, USA ISSN 0975 - 8887 Digital Library URI http://www.ijcaonline.org/archives/volume39/number 7/4829-7086

[18] Sunita B Aher and Lobo L.M.R.J. Article: Combination of Clustering, Classification \& Association Rule based Approach for Course Recommender System in E-learning. International Journal of Computer Applications 39(7):8-15, February 2012. Published by Foundation of Computer Science, New York, USA. ISSN 0975 - 8887 Digital Library URI: http://www.ijcaonline.org/archives/volume39/number 7/4830-7087

[19] Sunita B Aher and LOBO L.M.R.J.. Article: Prediction of Course Selection by Student using Combination of Data Mining Algorithms in ELearning. International Journal of Computer Applications 40(15):1-7, February 2012. Published by Foundation of Computer Science, New York, USA. ISSN 0975 - 8887 Digital Library URI http://www.ijcaonline.org/archives/volume40/number $15 / 5053-7085$ 